\title{
Inhibition of prostaglandin E2 protects abdominal aortic aneurysm from expansion through regulating miR-29b-mediated fibrotic ECM expression
}

\author{
ZONGLIN HAN ${ }^{1}$, TANGSHAN ZHANG ${ }^{2}$, YUXIANG HE ${ }^{1}$, GANG LI $^{1},{\text { GANG } \mathrm{LI}^{3} \text { and XING JIN }}^{1}$ \\ ${ }^{1}$ Department of Vascular Surgery, Shandong Provincial Hospital Affiliated to Shandong University, Jinan, Shandong 250021; \\ ${ }^{2}$ Department of Vascular Surgery, The Seventh People's Hospital, Jinan, Shandong 251400; \\ ${ }^{3}$ Department of General Surgery, Central Hospital of Taian, \\ Taian, Shandong 271000, P.R. China
}

Received January 4, 2018; Accepted May 8, 2018

DOI: $10.3892 /$ etm.2018.6160

\begin{abstract}
The risk of rupture, the most feared clinical consequence of abdominal aortic aneurysm, increases with the enlargement of aorta. MicroRNA-29b (miR-29b) has emerged as a key modulator of extracellular matrix (ECM) homeostasis and thereby is proposed to play a crucial role in vascular remodeling. However, agents that alter miR-29b expression are relatively inefficient in the aorta, likely due to inferior uptake. Herein we found that miR-29b was upregulated in aortic smooth muscle cells upon prostaglandin $\mathrm{E}_{2}\left(\mathrm{PGE}_{2}\right)$ stimulation whereas indomethacin treatment downregulated miR-29b expression. In order to obtain insight into the pathological processes associated with the vascular remodeling that accompanies aortic dilatation, we compared expression profiles of several representative ECM components in aortic walls. Notably, $\mathrm{PGE}_{2}$ induced a dramatic decline in these ECM components, which was rescued by introduction of indomethacin. In addition, COL1A1 was validated as a direct target gene of miR-29b by dual-luciferase reporter assay. In aggregate, our study suggests that $\mathrm{PGE}_{2}$ may accelerate ECM degradation through decreasing miR-29b expression. Thus those anti-inflammatory drugs that inhibit $\mathrm{PGE}_{2}$ synthesis represent an effective means of inducing an augmented profibrotic response in the aortic walls and thereby inhibiting aneurysmal expansion.
\end{abstract}

\section{Introduction}

An abdominal aortic aneurysm (AAA) occurs if the abdominal aorta becomes focally enlarged, resulting from a weakened

Correspondence to: Dr Xing Jin, Department of Vascular Surgery, Shandong Provincial Hospital Affiliated to Shandong University, 324 Jingwu Road, Jinan, Shandong 250021, P.R. China

E-mail: quu497484@163.com

Key words: fibrosis, abdominal aortic aneurysm, prostaglandin, microRNA-29b, extracellular matrix abdominal aortic wall. The prevalence of AAA has continued to increase worldwide over the last four decades. Data from clinical investigations involving different populations demonstrate that the prevalence is $2.4-16.9 \%$ in male and $0.5-2.2 \%$ in female patients older than 65 years of age (1). AAA usually develops asymptomatically until the outbreak of acute aneurysm rupture, which can cause severe internal bleeding and accounts for a mortality of $85-90 \%$ (2). The pathogenesis of AAA initiation, progression, and ultimate rupture has been rarely elucidated by previous studies. Dilatation and weakening of the aorta is accompanied by other phenotypic changes including local inflammation, smooth muscle cell apoptosis, oxidation stress increase and, in particularly, dramatic extracellular matrix (ECM) degradation (3).

MicroRNAs (miRNAs) are a class of endogenous noncoding RNAs encompassing 18-23 nucleotides that regulate the expression of mRNAs by targeting their 3'-untranslated regions (3'-UTRs) and inhibiting translation (4). Recently, miRNAs have been deemed as vital modulators of diverse cell events and have been implicated in a wide array of pathologies, such as cardiovascular disorders (5). Expression alterations in miRNAs have been revealed to impact the vascular angiogenesis, inflammation, and remodeling (6,7). As a member of the miR-29 family, microRNA-29b (miR-29b) has been previously proven to be remarkably upregulated in patients with AAA in comparison to the normal population (8). The miR-29 family can accelerate fibrosis in various tissues via regulating downstream ECM genes (9-11), including multiple collagens isoforms (COL1A1, COL5A1, COL3A1) and components of aortic wall, such as and elastin (ELN) and fibrillin-1 (FBN1). Dysregulation of ECM homeostasis is responsible for several pathological conditions, such as fibrosis and cancerous invasion. In addition, the aforementioned collagens that are widely expressed in the aortic wall play a vital role in aneurysm formation (12-14). Also, matrix metalloproteinases (MMPs, such as MMP2 and MMP9) have been identified as direct target genes of miR-29b and relate to AAA onset and progression $(15,16)$. These data in aggregate suggest that miR-29b may participate in AAA development via altering ECM microenvironment and suppressing ECM protein expression. 
Prostaglandins (PGs) are essentially a category of fatty acids that can result in inflammation, pain, redness and swelling. There are four major bioactive PGs that are ubiquitously generated in vivo and function as lipid mediators in autocrine and paracrine manner. Among them, prostaglandin E2 $\left(\mathrm{PGE}_{2}\right)$ is one of the most abundant PGs synthesized in the human body and possesses versatile physiological and/or pathological functions. While the pro-inflammatory property of $\mathrm{PGE}_{2}$ during acute inflammatory response is profoundly established, increasing studies have been launched with regard to its role in multiple vascular pathological conditions. For example, $\mathrm{PGE}_{2}$ induces augmentation of arterial dilatation and enhances microvascular permeability, thereby increasing blood flow into the inflamed tissues (17). On the other hand, $\mathrm{PGE}_{2}$ restrains the aortic smooth muscle cell (ASMC) proliferation and decreases cytokine secretion in vitro (18).

Prior studies have also shown that $\mathrm{PGE}_{2}$ is abundantly produced in the aneurysm wall, which may exert inhibitory effects on collagen synthesis $(19,20)$. In addition, $\mathrm{PGE}_{2}$ is significantly implicated in vascular wall remodeling via the regulation of MMP activities in human AAA (21). It has been demonstrated that the miR-29 family members were obviously upregulated in trabecular meshwork cells by exogenous $\mathrm{PGE}_{2}$-evoked stimuli (22). Fortunately we found that the expression of miR-29b in the ASMCs was elevated on $\mathrm{PGE}_{2}$ treatment in our tentative trial, justifying the assumption that $\mathrm{PGE}_{2}$ improves miR-29b-mediated ECM remodeling in AAA development.

\section{Materials and methods}

Cell culture. The Ethics Committee of the Provincial Hospital Affiliated to Shandong University approved the study (Jinan, China). Human ASMCs (passage no. 3) propagated in growth media SmGM-2 were both purchased from Lonza (Walkersville, MD, USA) supplemented with 5\% fetal bovine serum (FBS) following the manufacturer's instructions. $\mathrm{PGE}_{2}$ and indomethacin were purchased from Cayman Chemical (Ann Arbor, MI, USA). Cells were treated with $500 \mathrm{ng} / \mathrm{ml}$ $\mathrm{PGE}_{2}$ or $10 \mathrm{mmol} / \mathrm{l}$ indomethacin, with DMSO employed as a control. Cell containing plates were harvested for RNA or protein analysis at $\sim 90 \%$ confluence.

In particular, indomethacin solution was first prepared by dropwise addition of $1 \mathrm{~mol} / 1 \mathrm{Na}_{2} \mathrm{CO}_{3}$ to the drug powder until dissolved, and afterwards DMSO was added to make the solution concentration of $10.0 \mathrm{mmol} / \mathrm{l}$, followed by sterile filtering.

Transfection of cultured cells. The ASMCs were transfected with miRNA-29b mimic, inhibitor or Scr-miR (Dharmacon, Chicago, IL, USA) using Lipofectamine 2000 (Invitrogen, Burlington, ON, Canada). miRNA transfection efficiency was confirmed by RT-qPCR. Two hours after transfection, cells were treated with $\mathrm{PGE}_{2}$ or indomethacin for $24 \mathrm{~h}$ before they were harvested.

miRNA extraction and quantification. miRNAs were extracted from cells using the mirVana miRNA isolation kit (Ambion, Austin, TX, USA). Briefly, the cell samples were collected and washed two times using PBS, prior to the addition of miRNA additive (1:10) on ice for $15 \mathrm{~min}$. The cell lysate was added with equal volumes of acid-phenol:chloroform, before centrifugation and removal of the aqueous phase, and then the mixture was added 1.25 -fold to $100 \%$ ethanol. The mixture was passed through the filter cartridge and eluted. RT-qPCR was carried out with a final reaction volume of $20 \mathrm{ml}$ containing $10 \mathrm{ml}$ TaqMan Universal PCR Master Mix (Applied Biosystems; Thermo Fisher Scientific Inc., Waltham, MA, USA), 8 ml DEPC-treated water, $1 \mathrm{ml}$ TaqMan microRNA assay (Applied Biosystems; Thermo Fisher Scientific Inc.), and $1 \mathrm{ml} \mathrm{RT}$ product. The data were normalized to RNU6B small nuclear RNA to calculate fold-changes using the method of $\Delta \Delta \mathrm{Cq}$.

Dual-luciferase reporter assay. Two online databases, miRBase and TargetScan, were used to predict the potential binding sites for miR-29b. For dual-luciferase reporter assays, the full-length 3'-UTR of COL1A1 containing three miR-29b binding sites was cloned into the downstream of a pMIR-Report (Ambion) to generate pMir-COL1A1 3'-UTR, which was co-transfected with miR-29b mimics or Scr-miR into ASMCs. The pRL-SV40 vector (Promega, Madison, WI, USA) carrying the Renilla luciferase gene was used as an internal control to normalize the transfection efficiency. Luciferase activities were determined by using the Dual-Luciferase Reporter assay system (Promega) following the manufacturer's instructions. All the reactions were performed in triplicate.

mRNA quantification by RT-qPCR. Total RNA was isolated using TRIzol reagent (Invitrogen, Carlsbad, CA, USA) following the manufacturer's instructions. The total RNA $40 \mu \mathrm{g}$ was used as template and then reverse transcribed with M-MLV Reverse Transcriptase kit (Promega Biotech Co., Ltd, Beijing, China) to synthesize the cDNA. Expression levels of target genes were normalized to $\beta$-actin, the internal positive control. RT-qPCR was carried out in a LightCycler (Roche Diagnostics, Laval, QC, Canada) machine with the SYBR-Green probe (SYBR Premix Ex Taq ${ }^{\mathrm{TM}}$ II; Takara, Dalian, China). Melting-curve analysis was used to determine the melting temperature ( $\mathrm{Tm})$ of specific amplification products and primer dimers, which were used for the signal acquisition step $\left(2-3^{\circ} \mathrm{C}\right.$ below $\left.\mathrm{Tm}\right)$ for each gene. The comparative $\mathrm{Cq}(2-\Delta \Delta \mathrm{Cq})$ method was introduced to account for the relative fold-changes of the amount of template differences.

Protein extraction and western blotting. The harvested cells were pelleted and resuspended in RIPA lysis buffer, followed by incubating on ice for further lysing and centrifuged at $15,000 \mathrm{x} \mathrm{g}$ for $5 \mathrm{~min}$ at $4^{\circ} \mathrm{C}$. After centrifugation, protein supernatant was kept at $-80^{\circ} \mathrm{C}$ for future analysis.

For immunoblotting, $30 \mu \mathrm{g}$ of total protein was separated on $10 \%$ SDS-PAGE and transferred to PVDF membrane at $250 \mathrm{~mA}$ for $1 \mathrm{~h}$. The membrane was blocked with 5\% silk milk and then incubated overnight at $4^{\circ} \mathrm{C}$ with mouse monoclonal primary antibody mouse monoclonal anti-COL1A1 (sc-293182; 1:1,000; Santa Cruz Biotechnology, Inc., Santa Cruz, CA, USA), followed by subsequent incubation with HRP-conjugated secondary goat anti-mouse polyclonal IgG (SA132; 1:2,000; Beijing Solarbio Science and Technology Co., Ltd., Beijing, China) at room temperature for $1 \mathrm{~h}$. Then, the membrane was washed with PBST three times ( $5 \mathrm{~min} /$ time) before photographed using ECL reagents (Millipore, Billerica, MA, USA). 
Soluble collagen assay. The total soluble collagen secreted from ASMCs was evaluated following the manufacturer's instructions (QuickZyme Biosciences, Leiden, Netherlands). In brief, after treatment with $\mathrm{PGE}_{2}$ or indomethacin for $24 \mathrm{~h}$, companied with transfection with miR-29b mimics, inhibitor, or scr-miR, conditioned culture medium was collected and centrifuged to remove cell debris. Samples were incubated with Sirius Red color dye for $10 \mathrm{~min}$ at room temperature. After precipitation in a 96-well plate, data analysis was performed on a microplate reader (Multiskan MK3; Thermo Labsystems, Franklin, MA, USA) based on the absorbance at $540 \mathrm{~nm}$. The experiment was performed in triplicate.

Statistical analysis. Data are presented as means \pm SD. All in vitro experiments included at least 3 replicates per group. Groups were compared using the two-tailed Student's t-test for parametric data. When comparing multiple groups, data were analyzed by ANOVA with Bonferroni's post-hoc test. $\mathrm{P}<0.05$ was considered to indicate a statistically significant difference.

\section{Results}

miR-29b expression was significantly increased on $P G E_{2}$ treatment. In our earlier study, we found that upon $\mathrm{PGE}_{2}$ incitement, miR-29b was significantly upregulated in ASMCs, unusual undifferentiated muscle cell possessing the capacity to produce ECM. In order to further confirm this, ASMCs were treated with $500 \mathrm{ng} / \mathrm{ml} \mathrm{PGE}_{2}$ or $10 \mathrm{mmol} / \mathrm{l}$ indomethacin, a non-steroidal anti-inflammatory drug (NSAID), in prior to the evaluation of miR-29b expression in vitro (Fig. 1). Strictly consistent with previous results, miR-29b was dramatically increased in $\mathrm{PGE}_{2}$-treated ASMCs compared to untreated cells $(2.162 \pm 0.117$ vs. $1.004 \pm 0.010, P<0.001)$, whereas miR-29b was significantly downregulated in the presence of indomethacin $(0.665 \pm 0.015$ vs. $1.004 \pm 0.010$, when compared with untreated cells; $\mathrm{P}<0.01)$.

miR-29b directly targets the 3'-UTR of COL1Al gene in cultured SVMCs. Although various ECM components have been identified as targets of miR-29b in other tissues, the downstream target genes in ASMCs remain unknown. As shown in Fig. 2A, the putative miR-29 binding sites are enriched in the 3'-UTR region of COL1A1 gene. To confirm whether miR-29b could directly target COL1A1, the 3'-UTR region of COL1A1 gene was cloned into a luciferase reporter vector, which was co-transfected into ASMCs with the miR-29b mimics or Scr-miR, prior to the performance of the luciferase reporter assay. In the presence of miR-29b mimics, the relative luciferase activity of COL1A1-3'-UTR-transfected ASMCs was significantly decreased compared with those untransfected cell control (Fig. 2B), whereas Scr exposure had no significant effect on the fluorescence intensity of the COL1A1-3'-UTR-transfected ASMCs.

We next assessed the effect of miR-29b on the COL1A1 expression level in ASMCs by using RT-qPCR assay. The results demonstrated that the expression of COL1A1 in miR-29b mimics-transfected ASMCs was reduced at mRNA level compared to untransfected cells (Fig. 3A;

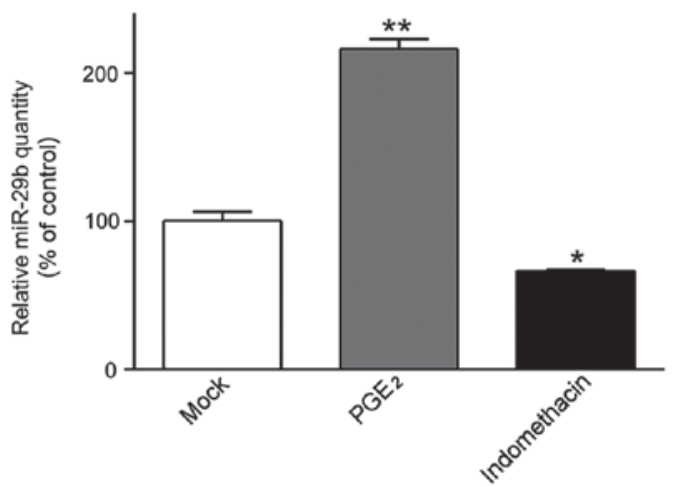

Figure 1. miR-29b expression was significantly increased on $\mathrm{PGE}_{2}$ treatment. AMSCs were treated with $\mathrm{PGE}_{2}$ or indomethacin. After $24 \mathrm{~h}, \mathrm{miR}-29 \mathrm{~b}$ were isolated from cells and subjected to RT-qPCR assay for quantification. miR-29b expression in untreated cells were defined as $100 \%$. All data were presented as mean \pm standard deviation. ${ }^{*} \mathrm{P}<0.01$ and ${ }^{* *} \mathrm{P}<0.001$. $\mathrm{PGE}_{2}$, prostaglandin $\mathrm{E}_{2}$; AMSCs, aortic smooth muscle cells.

$0.587 \pm 0.178$-fold, $\mathrm{P}<0.05$ ), which was corroborated by immunoblotting assay (Fig. 2C). These results suggest that miR-29b exerts an inhibitory effect on COL1A1 expression through directly targeting the $3^{\prime}$-UTR.

PGE2 promotes miR-29b-mediated ECM degradation in $A S M C s$. It has been reported that $\mathrm{PGE}_{2}$ participates in vascular wall remodeling via modulating MMP activities (21), thus we speculated whether $\mathrm{PGE}_{2}$ is able to regulate other profibrosisassociated ECM components. Collagen gene expression alterations (represented by COL1A1 and COL3A1) in AMSCs were assessed in the presence of $\mathrm{PGE}_{2}$. As shown in Fig. 3A, upon $\mathrm{PGE}_{2}$ treatment, the mRNA expression of COL1A1 and COL3A1 was significantly repressed $(0.690 \pm 0.193$ and $0.710 \pm 0.145$-fold, $\mathrm{P}<0.05$, respectively). Likewise, $\mathrm{PGE}_{2}$ also inhibited the expression of ELN and FBN1 in ASMCs (0.657 \pm 0.105 -fold and $0.643 \pm 0.235$-fold, $\mathrm{P}<0.01$, respectively). Of note, ECM levels were augmented in response to indomethacin.

To further investigate whether $\mathrm{PGE}_{2}$ exerts inhibitory effects on ECM expression via upregulating miR-29b, miR-29b in ASMCs was then suppressed by transfecting with its inhibitor, followed by assessing the ECM expression profiles. Successful inhibition of miR-29b ( $<30 \%$ of expression), regardless of $\mathrm{PGE}_{2}$ treatment, was confirmed by RT-qPCR analysis (data not shown). Interestingly, expression levels of ECM genes in $\mathrm{PGE}_{2}$-stimulated and miR-29b inhibitor-transfected ASMCs were not inhibited but even increased compared to the untreated control (Fig. 3A). These collectively indicated that $\mathrm{PGE}_{2}$ regulates ECM production by altering miR-29 expression.

The promoted effect of $\mathrm{PGE}_{2}$ on miR-29b-mediated ECM downregulation in ASMCs was re-confirmed by monitoring soluble collagen synthesis after incitement. As shown in Fig. 3B, soluble collagen production was decreased in $\mathrm{PGE}_{2}$-treated ASMCs in comparison to that in untreated control. Notably, transfection of miR-29b inhibitor significantly compromised the inhibitory effect of $\mathrm{PGE}_{2}$ upon soluble collagen synthesis, whereas miR-29b mimics exacerbated ECM degradation caused by $\mathrm{PGE}_{2}$ treatment. 

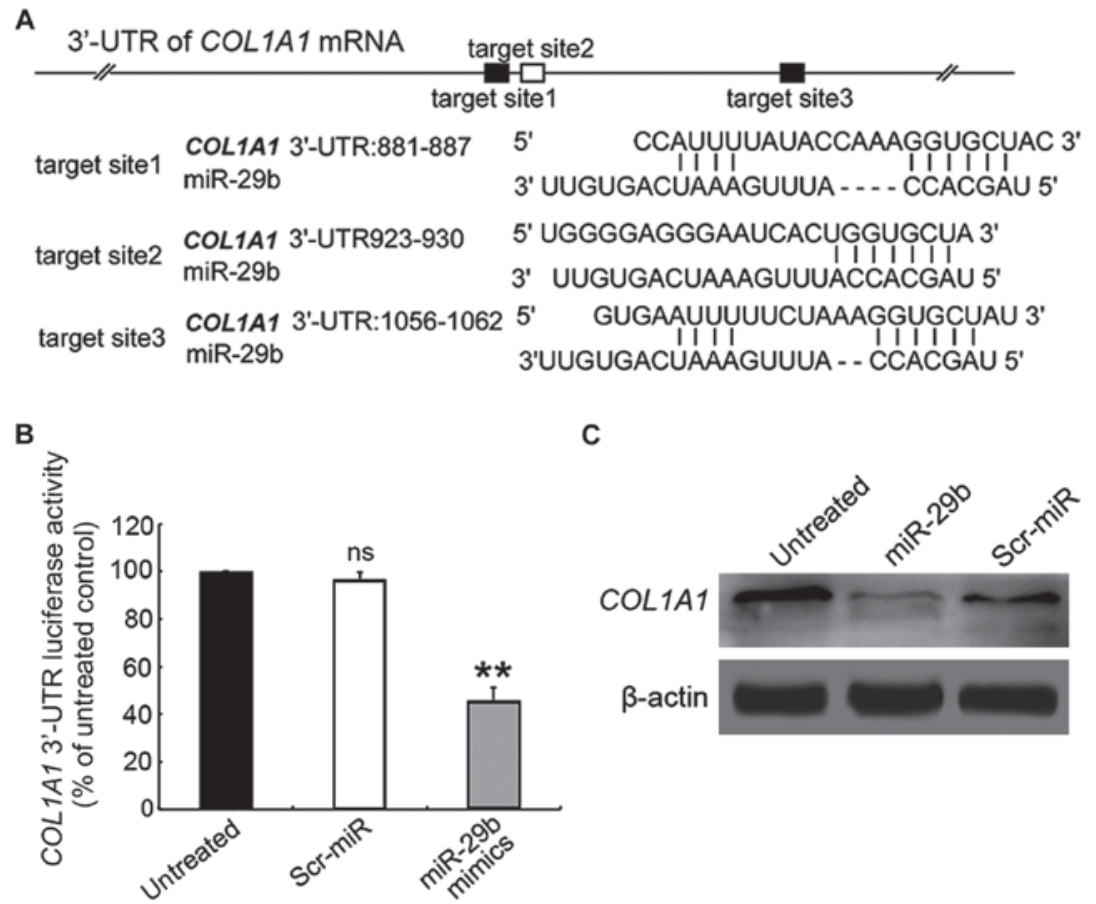

C

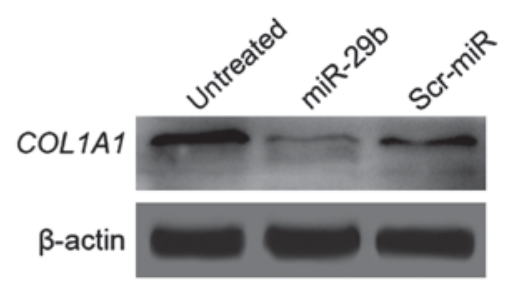

Figure 2. COL1A1 is a direct target of miR-29b. (A) The full-length 3'-UTR of COL1A1 mRNA contains three miR-29b-binding sites. (B) ASMCs were co-transfected with either miR-29b mimics or Scr-miR control, together with COL1A1-3'-UTR sequence. The relative luciferase activities were measured and normalized by Renilla luciferase value after $72 \mathrm{~h}$. All data are presented as mean \pm standard deviation. Similar results were obtained from 3 independent experiments. ${ }^{* *} \mathrm{P}<0.01$. (C) COL1A1 protein level in ASMCs cells co-transfected with either miR-29b mimics or Scr-miR control. 3'-UTR, 3'-untranslated region; ASMCs, aortic smooth muscle cells.

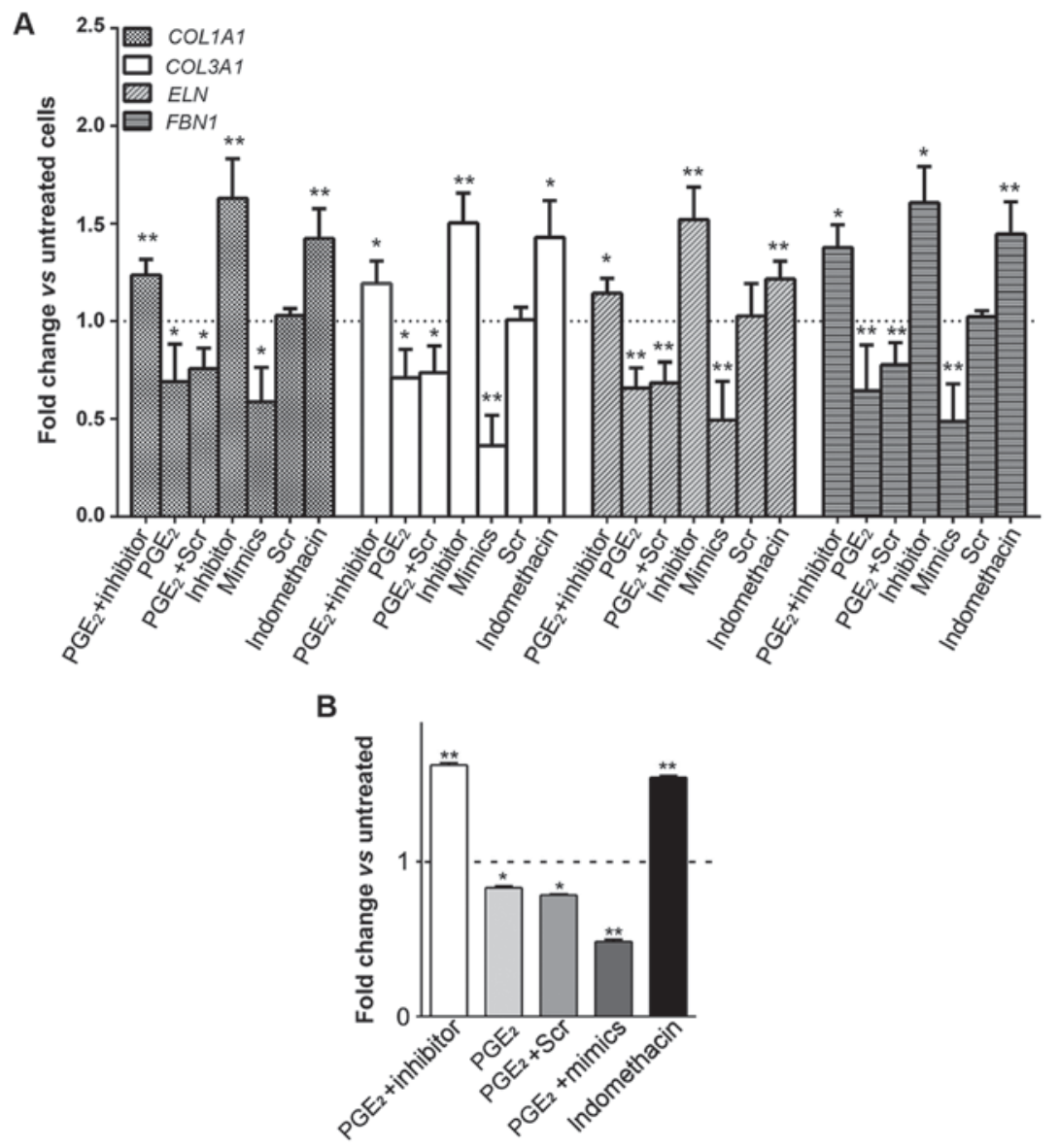

Figure 3. Alteration in the expression profiles of ECM components under different conditions. (A) Expression levels of ECM genes in ASMCs were assessed by RT-qPCR. (B) Soluble collagens produced in ASMCs. Data are presented as mean \pm standard deviation. ${ }^{*} \mathrm{P}<0.05,{ }^{* *} \mathrm{P}<0.01$ vs. untreated cells. ECM, extracellular matrix; ASMCs, aortic smooth muscle cells. 


\section{Discussion}

The pathogenesis of an aneurysm as well as its progression and ultimate rupture involve series of complicated pathological processes. The determination of underlying mechanisms and identification of effective medical therapy remains a major challenge in recent aneurysmal medicine. In particular, novel molecular therapies in conquering AAA seem to be of vital importance. As far, patients with larger aneurysms depend on elective surgery. Understanding the miR-mediated regulation of ECM perturbations in pathological conditions will potentially provide insightful prospective in seeking innovative medical strategies to fight AAA.

In a separate case-control study, the expansion of AAAs in patients taking NSAIDs was significantly repressed compared with the control subjects (18), suggesting that inhibition of PGs synthesis in AAA development. PGs are ubiquitously generated in all cell types and function as autocrine and paracrine regulators to maintain local homeostasis or inflammatory response. Among them, $\mathrm{PGE}_{2}$ is the most prevalent and bioactive of the mammalian PG, which was found previously to be abundantly generated in the aneurysm wall and involved in the regulation of collagen synthesis (19). It has been demonstrated that during viral infection, there was a dramatic increase in the expression level of miR-29, which inhibited DNA methyl transferase (DNMT) activity and thereby contribute to the activation of $\mathrm{COX} 2$ and consequent enhancement of $\mathrm{PGE}_{2}$ production (23). Collectively, we conclude that miR-29 promotes $\mathrm{PGE}_{2}$ accumulation in response to inflammation through epigenetic modification-induced COX2 activation. However the effects of PGE $_{2}$ on miR-29 and the sequential ECM-mediated inflammatory signaling pathways have rarely been studied.

In this study, we investigated the underlying mechanism of $\mathrm{PGE}_{2}$ and the pharmacology of its blockade, by introduction of an NSAID indomethacin, in orchestrating the inflammatory response, with particular regard to the AAA. Data from our experiments indicated that treatment of ASMCs with $\mathrm{PGE}_{2}$ induced an increase in miR-29b level, whereas indomethacin resulted in a decrease in miR-29b expression, hence a profibrotic response in AAA.

The miR-29 family is well characterized by its capacity to inhibit the expression of ECM components and thereby block the related fibrosis in a variety of organs. Previously published studies have already manifested the therapeutic implication of miR-29 as a target for fibrosis in heart (11), lung (24), liver (25), and kidney (26), and systemic sclerosis (27). In fibrotic conditions, decreased miR-29b directly results in an aggrandized collagen gene expression. Unfortunately, a profibrotic response in these organs is often pathologic and related to several serious diseases. Whether miR-29b downregulation plays a beneficial role in conquering certain fibrosis-related diseases is not known Although the precise mechanisms remain unclear, repression of miR-29b expression in experimental AAA development has been widely reported in previously published studies (9-11). Fig. 3 shows that modulation of miR-29b levels in ASMCs, especially overexpression with miR-29b mimics or suppression by miR-29b inhibitor, resulted in significant alteration in expression profiles of target collagen genes. Treatment of smooth muscle cells with $\mathrm{PGE}_{2}$ elevated miR-29b expression and thereby suppressed the ECM genes expression compared to untreated cells, while an inhibitor of PG synthesis termed as indomethacin counteracts the promotion by $\mathrm{PGE}_{2}$ of $\mathrm{ECM}$ genes expression. Of note, introduction of miR-29b inhibitor compromised the effects of $\mathrm{PGE}_{2}$ on collagen gene expression profiles, suggesting that $\mathrm{PGE}_{2}$ plays an inhibitory role in ECM expression by targeting miR-29b.

Taken together, we proposed that there is a novel bidirectional positive-feedback loop between $\mathrm{PGE}_{2}$ synthesis and miR-29b, functioning as a node in the regulation of ECM homeostasis. When AAA disease occurs, miR-29b expression level is obviously elevated, which inhibits methylation degree of COX2 and enhances its activity, and this will ultimately promote $\mathrm{PGE}_{2}$ accumulation. On the contrary, increased $\mathrm{PGE}_{2}$ can further accelerate miR-29b augmentation and therefore induce the pathological degradation of ECM proteins.

Data from a previous study suggested that a decline in the miR-29b expression triggers a profibrotic process in AAAs (28), which is usually deemed as a pathologic response to aneurismal dilatation. As the aneurysms dilate is companied by an increase in the risk of rupture, the aortic wall may tend, like a balloon, to become thinner and weaker compared to smaller aneurysms. In this case, deposition of new more soluble collagen fibers in aortic wall, leading to the thickening of aneurysmal walls, will occur to compensate the attenuated media and to reduce arterial wall tension (29). Although the new generated soluble collagens are more susceptible to hydrolysis by MMPs, the fibrotic thickening of the adventitia will hinder aneurysm expansion. Our observations highlighted the extent of soluble collagens degradation that took place in ASMCs as exposed to $\mathrm{PGE}_{2}$ treatment, which was rescued by introduction of indomethacin. Furthermore, upon $\mathrm{PGE}_{2}$ treatment, there was a decline in ELN content, which is synthesized constitutively by smooth muscle cells in the media. Since the destruction of ELN fibers has been suggested as a significant pathological process in aortic dilatation and being related to the loss of elastic capacities of the aortic media $(30,31)$, the above suggest that $\mathrm{PGE}_{2}$ as well as miR-29b are crucially involved in the aneurysmal expansion. However, the application of agents that modulate miR-29b expression is relatively less efficient in the aorta compared to that in other organs such as kidney, liver and heart (28), likely due to preferential uptake in these organs. In this study, drugs targeting $\mathrm{PGE}_{2}$ are potentially more effective in control of aneurysmal dilatation.

In conclusion, in this study we propose that more expandable anti-inflammatory drugs that inhibit $\mathrm{PGE}_{2}$ synthesis may emerge as a promising avenue to trigger fibrosis in the aortic wall and thereafter protect the aorta from expansion in human patients with AAA. The in vivo experiments underlying their therapeutic potential to inhibit aneurysm expansion and ultimate rupture are being carried out in our laboratory and further evidence will be published in the future.

\section{Acknowledgements}

Not applicable.

\section{Funding}

This study did not receive any specific grant from funding agencies in the public, commercial, or not-for-profit sectors. 


\section{Availability of data and materials}

The datasets used and/or analyzed during the present study are available from the corresponding author on reasonable request.

\section{Authors' contributions}

$\mathrm{ZH}$ and $\mathrm{XJ}$ contributed to the conception of the study. TZ contributed significantly to the data analysis and study preparation. YH and GL performed the data analyses and wrote the study. GL helped perform the analysis with constructive discussions. All authors have read and approved the final study.

\section{Ethics approval and consent to participate}

The Ethics Committee of the Provincial Hospital Affiliated to Shandong University approved the study (Jinan, China).

\section{Consent for publication}

Not applicable.

\section{Competing interests}

All authors declare that they have no financial or other conflicts of interest in relation to this study and its publication.

\section{References}

1. Weintraub NL: Understanding abdominal aortic aneurysm. N Engl J Med 361: 1114-1116, 2009.

2. Kent KC: Clinical practice. Abdominal aortic aneurysms. N Engl J Med 371: 2101-2108, 2014.

3. Thompson RW, Liao S and Curci JA: Vascular smooth muscle cell apoptosis in abdominal aortic aneurysms. Coron Artery Dis 8: 623-631, 1997.

4. Zhang C: MicroRNomics: A newly emerging approach for disease biology. Physiol Genomics 33: 139-147, 2008.

5. Seeger T and Boon RA: MicroRNAs in cardiovascular ageing. J Physiol 594: 2085-2094, 2016.

6. Small EM and Olson EN: Pervasive roles of microRNAs in cardiovascular biology. Nature 469: 336-342, 2011.

7. Urbich C, Kuehbacher A and Dimmeler S: Role of microRNAs in vascular diseases, inflammation, and angiogenesis. Cardiovasc Res 79: 581-588, 2008.

8. Kin K, Miyagawa S, Fukushima S, Shirakawa Y, Torikai K, Shimamura K, Daimon T, Kawahara Y, Kuratani T and Sawa Y: Tissue- and plasma-specific microRNA signatures for atherosclerotic abdominal aortic aneurysm. J Am Heart Assoc 1: e000745, 2012.

9. Roderburg C,Urban GW, Bettermann K, Vucur M,Zimmermann H, Schmidt S, Janssen J, Koppe C, Knolle P, Castoldi M, et al: Micro-RNA profiling reveals a role for miR-29 in human and murine liver fibrosis. Hepatology 53: 209-218, 2011.

10. Qin W, Chung AC, Huang XR, Meng XM, Hui DS, Yu CM, Sung JJ and Lan HY: TGF- $\beta / \mathrm{Smad} 3$ signaling promotes renal fibrosis by inhibiting miR-29. J Am Soc Nephrol 22: 1462-1474, 2011.

11. Van Rooij E, Sutherland LB, Thatcher JE, DiMaio JM, Naseem RH, Marshall WS, Hill JA and Olson EN: Dysregulation of microRNAs after myocardial infarction reveals a role of miR-29 in cardiac fibrosis. Proc Natl Acad Sci USA 105 13027-13032, 2008.

12. De Paepe A, Nuytinck L, Hausser I, Anton-Lamprecht I and Naeyaert JM: Mutations in the COL5A1 gene are causal in the Ehlers-Danlos syndromes I and II. Am J Hum Genet 60: 547-554, 1997.

13. Rahkonen O, Su M, Hakovirta H, Koskivirta I, Hormuzdi SG, Vuorio E, Bornstein P and Penttinen R: Mice with a deletion in the first intron of the Collal gene develop age-dependent aortic dissection and rupture. Circ Res 94: 83-90, 2004.
14. Menashi S, Campa JS, Greenhalgh RM and Powell JT: Collagen in abdominal aortic aneurysm: Typing, content, and degradation. J Vasc Surg 6: 578-582, 1987.

15. Rizzo RJ, McCarthy WJ, Dixit SN, Lilly MP, Shively VP, Flinn WR and Yao JS: Collagen types and matrix protein content in human abdominal aortic aneurysms. J Vasc Surg 10: 365-373, 1989.

16. Chen KC, Wang YS, Hu CY, Chang WC, Liao YC, Dai CY and Juo SH: OxLDL up-regulates microRNA-29b, leading to epigenetic modifications of MMP-2/MMP-9 genes: A novel mechanism for cardiovascular diseases. FASEB J 25: 1718-1728, 2011.

17. Funk CD: Prostaglandins and leukotrienes: Advances in eicosanoid biology. Science 294: 1871-1875, 2001.

18. Walton LJ, Franklin IJ, Bayston T, Brown LC, Greenhalgh RM, Taylor GW and Powell JT: Inhibition of prostaglandin E2 synthesis in abdominal aortic aneurysms: Implications for smooth muscle cell viability, inflammatory processes, and the expansion of abdominal aortic aneurysms. Circulation 100: 48-54, 1999.

19. Diaz A, Munoz E, Johnston R, Korn JH and Jimenez SA: Regulation of human lung fibroblast alpha 1(I) procollagen gene expression by tumor necrosis factor alpha, interleukin-1 beta, and prostaglandin E2. J Biol Chem 268: 10364-10371, 1993.

20. Holmes DR, Wester W, Thompson RW and Reilly JM: Prostaglandin E2 synthesis and cyclooxygenase expression in abdominal aortic aneurysms. J Vasc Surg 25: 810-815, 1997.

21. Yokoyama U, Ishiwata R, Jin MH, Kato Y, Suzuki O, Jin H, Ichikawa Y, Kumagaya S, Katayama Y, Fujita T, et al: Inhibition of EP4 signaling attenuates aortic aneurysm formation. PLoS One 7: e36724, 2012.

22. Gonzalez P, Luna C, Li G, Qiu J and Epstein DL: miR-29 is induced by prostaglandin $\mathrm{E}$ and forms negative feedback loops with the Wnt and TGFbeta pathways in human trabecular meshwork cells. Invest Ophthalmol Vis Sci 51: 3210, 2010.

23. Fang J, Hao Q, Liu L, Li Y, Wu J, Huo X and Zhu Y: Epigenetic changes mediated by microRNA miR29 activate cyclooxygenase 2 and lambda-1 interferon production during viral infection. J Virol 86: 1010-1020, 2012.

24. Xiao J, Meng XM, Huang XR, Chung AC, Feng YL, Hui DS, Yu CM, Sung JJ and Lan HY: miR-29 inhibits bleomycin-induced pulmonary fibrosis in mice. Mol Ther 20: 1251-1260, 2012.

25. Zhang Y, Wu L, Wang Y, Zhang M, Li L, Zhu D, Li X, Gu H, Zhang CY and Zen K: Protective role of estrogen-induced miRNA-29 expression in carbon tetrachloride-induced mouse liver injury. J Biol Chem 287: 14851-14862, 2012.

26. Wang B, Komers $\mathrm{R}$, Carew $\mathrm{R}$, Winbanks CE, Xu B, Herman-Edelstein M, Koh P, Thomas M, Jandeleit-Dahm K, Gregorevic P, et al: Suppression of microRNA-29 expression by TGF- $\beta 1$ promotes collagen expression and renal fibrosis. J Am Soc Nephrol 23: 252-265, 2012.

27. Maurer B, Stanczyk J, Jüngel A, Akhmetshina A, Trenkmann M, Brock M, Kowal-Bielecka O, Gay RE, Michel BA, Distler JH, et al: MicroRNA-29, a key regulator of collagen expression in systemic sclerosis. Arthritis Rheum 62: 1733-1743, 2010.

28. Maegdefessel L, Azuma J, Toh R, Merk DR, Deng A, Chin JT, Raaz U, Schoelmerich AM, Raiesdana A, Leeper NJ, et al: Inhibition of microRNA-29b reduces murine abdominal aortic aneurysm development. J Clin Invest 122: 497-506, 2012.

29. Sakalihasan N, Heyeres A, Nusgens BV, Limet R and Lapière CM: Modifications of the extracellular matrix of aneurysmal abdominal aortas as a function of their size. Eur J Vasc Surg 7: 633-637, 1993.

30. Martufi G, Satriano A, Moore RD, Vorp DA and Di Martino ES: Local quantification of wall thickness and intraluminal thrombus offer insight into the mechanical properties of the aneurysmal aorta. Ann Biomed Eng 43: 1759-1771, 2015.

31. Hance KA, Tataria M, Ziporin SJ, Lee JK and Thompson RW: Monocyte chemotactic activity in human abdominal aortic aneurysms: Role of elastin degradation peptides and the 67-kD cell surface elastin receptor. J Vasc Surg 35: 254-261, 2002.

This work is licensed under a Creative Commons Attribution-NonCommercial-NoDerivatives 4.0 International (CC BY-NC-ND 4.0) License. 